\title{
Lattice QCD Study of Transverse-Momentum Dependent Soft Function
}

\author{
Yuan Li॰, ${ }^{1}$ Shi-Cheng Xia, ${ }^{1}$ Constantia Alexandrou, ${ }^{2,3}$ Krzysztof Cichy, ${ }^{4}$ Martha Constantinou $\odot,{ }^{5}$ \\ Xu Feng $\odot,{ }^{1,6,7, *}$ Kyriakos Hadjiyiannakou, ${ }^{3}$ Karl Jansen, ${ }^{8}$ Chuan Liu ${ }^{1,6,7}$ Aurora Scapellato, ${ }^{5}$ \\ Fernanda Steffens, ${ }^{9}$ and Jacopo Tarello ${ }^{3}$ \\ ${ }^{1}$ School of Physics and State Key Laboratory of Nuclear Physics and Technology, Peking University, Beijing 100871, China \\ ${ }^{2}$ Department of Physics, University of Cyprus, P.O. Box 20537, 1678 Nicosia, Cyprus \\ ${ }^{3}$ Computation-based Science and Technology Research Center, The Cyprus Institute, 20 Kavafi Str., Nicosia 2121, Cyprus \\ ${ }^{4}$ Faculty of Physics, Adam Mickiewicz University, ul. Uniwersytetu Poznańskiego 2, 61-614 Poznań, Poland \\ ${ }^{5}$ Temple University, 1925 N. 12th Street, Philadelphia, Pennsylvania 19122-1801, USA \\ ${ }^{6}$ Collaborative Innovation Center of Quantum Matter, Beijing 100871, China \\ ${ }^{7}$ Center for High Energy Physics, Peking University, Beijing 100871, China \\ ${ }^{8}$ NIC, DESY, Platanenallee 6, D-15738 Zeuthen, Germany \\ ${ }^{9}$ Institut für Strahlen- und Kernphysik, Rheinische Friedrich-Wilhelms-Universität Bonn, \\ Nussallee 14-16, 53115 Bonn, Germany
}

(Received 25 June 2021; revised 22 November 2021; accepted 24 January 2022; published 8 February 2022)

\begin{abstract}
In this work, we perform a lattice QCD study of the intrinsic, rapidity-independent soft function within the framework of large momentum effective theory. The computation is carried out using a gauge ensemble of $N_{f}=2+1+1$ clover-improved twisted mass fermion. After applying an appropriate renormalization procedure and the removal of significant higher-twist contamination, we obtain the intrinsic soft function that is comparable to the one-loop perturbative result at large external momentum. The determination of the nonperturbative soft function from first principles is crucial to sharpen our understanding of the processes with small transverse momentum such as the Drell-Yan production and the semi-inclusive deep inelastic scattering. Additionally, we calculate the Collins-Soper evolution kernel using the quasi-transverse-momentumdependent wave function as input.
\end{abstract}

DOI: 10.1103/PhysRevLett.128.062002

Introduction.-Understanding the structure of matter within the framework of quantum chromodynamics (QCD) is one of the central goals of hadron and nuclear physics. Although the study of partonic transverse momentum dependent (TMD) phenomena started few years after QCD was proposed [1], our knowledge of TMD parton distribution functions (TMDPDFs) is still limited, both experimentally and theoretically (see, e.g., Refs. [2,3]) since, until recently, a systematic $a b$ initio computation of TMDPDFs was out of reach.

Knowledge of TMDPDFs would open a new window in our understanding of hadron structure, arising, for instance, from probing the coupling of $k_{\perp}$ of a given quark with its spin [4]. However, these functions cannot be obtained from totally inclusive processes, as we need an observable in the final state carrying information on $k_{\perp}$, obtained, e.g., by measuring the transverse momentum $Q_{\perp}$ of a lepton pair produced in a Drell-Yan process. Consequently, they are

Published by the American Physical Society under the terms of the Creative Commons Attribution 4.0 International license. Further distribution of this work must maintain attribution to the author(s) and the published article's title, journal citation, and DOI. Funded by SCOAP . intrinsically harder to measure. Nevertheless, the future Electron-Ion Collider in the U.S. [5] and that in China [6] have as one of their goals to make precise measurements of TMDPDFs, aiming to reconstruct a three-dimensional picture of hadrons in momentum space. As in the case of collinear PDFs, the extraction of TMDPDFs from the measured Drell-Yan or semi-inclusive deep inelastic cross sections is possible, thanks to factorization theorems, which isolate the nonperturbative physics into suitable definitions of TMDPDFs [7-11]. Unfortunately, for distributions dependent on $k_{\perp}$ there appears an extra divergence associated with the emission of gluons carrying small momenta, which is not canceled by the real and virtual perturbative corrections. These divergences are encoded into functions called soft functions. At large transverse momentum $Q_{\perp} \gg \Lambda_{\mathrm{QCD}}$, the soft function can be calculated using perturbation theory $[12,13]$. However, when the soft function captures the soft-gluon effects at small $Q_{\perp}$, it is generically nonperturbative.

Recently, using large momentum effective theory (LaMET) [14-16] a novel method has been proposed to extract the soft function from pion matrix elements (MEs) [17] that can be calculated in lattice QCD, enabling a solution of the difficult problem of nonperturbatively 
determining the soft function. A first exploratory lattice QCD calculation was carried out by the LPC Collaboration [18]. However, a better understanding of the new method with a deeper examination of various systematic aspects involved in a lattice calculation is important in order to further establish the validity of the approach.

In this work, we perform a calculation of the soft function using a different fermionic discretization, namely, the twisted mass fermion. We demonstrate the validity of the methodology proposed by Ref. [17] and determine the soft function, showing that to obtain the final results requires highly nontrivial steps including the following: (i) We apply an appropriate renormalization procedure [19-21] to remove power and logarithmic divergences in the nonlocal operators; (ii) we examine various pion MEs and find that some of the so-called higher-twist (HT) contaminations are substantial and can even flip the sign of the MEs. By designing improved pion MEs to cancel the HT effects, we show that we can reliably obtain the soft function; (iii) we perform the calculation at four different pion masses in order to examine the mass dependence of the soft function; (iv) we perform a detailed investigation of excited states; and (v) we examine its convergence when the external momentum increases.

An important additional component of this work is the calculation of the Collins-Soper evolution kernel, where we find results that are in qualitative agreement with other lattice QCD calculations [18,22,23].

Theoretical framework-As proposed in Ref. [17], the intrinsic, rapidity-independent soft function $S\left(b_{\perp}, \mu\right)$ depends on the transverse separation $b_{\perp}$ and the renormalization scale $\mu$. Using LaMET, it can be extracted from the pion $\operatorname{ME~} F_{\Gamma}\left(b_{\perp}, P^{z}\right)$, which is defined in Euclidean spacetime as [17]

$$
F_{\Gamma}\left(b_{\perp}, P^{z}\right)=\left\langle\pi\left(-P^{z}\right)\left|\bar{u} \Gamma u\left(b_{\perp}\right) \bar{d} \Gamma d(0)\right| \pi\left(P^{z}\right)\right\rangle .
$$

Here, $P^{z}$ is a large momentum in the $z$ direction carried by the pion. Two current operators $\bar{u} \Gamma u$ and $\bar{d} \Gamma d$ are inserted at the same time slice, but with a spatial separation $b_{\perp}$ that is perpendicular to the momentum direction. To extract the leading-twist (LT) contribution, one can choose the Dirac matrices as $\Gamma=I, \gamma_{5}, \gamma_{\perp}$ or $\gamma_{5} \gamma_{\perp} . F_{\Gamma}\left(b_{\perp}, P^{z}\right)$ can be factorized into the quasi-TMD wave function (quasiTMDWF) $\Phi$ and the intrinsic soft function $S\left(b_{\perp}, \mu\right)$ [14,17] at large $P^{z}$ through

$$
\begin{gathered}
F_{\Gamma}\left(b_{\perp}, P^{z}\right) \stackrel{P^{z} \rightarrow \infty}{=} S\left(b_{\perp}, \mu\right) \int_{0}^{1} d x d x^{\prime} H_{\Gamma}\left(x, x^{\prime}, P^{z}, \mu\right) \\
\quad \times \Phi^{\dagger}\left(x^{\prime}, b_{\perp},-P^{z}\right) \Phi\left(x, b_{\perp}, P^{z}\right),
\end{gathered}
$$

where $H_{\Gamma}\left(x, x^{\prime}, P^{z}, \mu\right)$ is the perturbative hard kernel. The quasi-TMDWF $\Phi$ is defined as

$\Phi\left(x, b_{\perp}, \pm P^{z}\right)=\lim _{l \rightarrow \infty} \int \frac{d \xi}{2 \pi} e^{i x \xi} \phi\left( \pm z, b_{\perp}, \pm l, \pm P^{z}\right)$ with $\xi=z P^{z}$. The wave function $\phi$ is given by

$$
\phi\left(z, b_{\perp}, l, P^{z}\right)=\left\langle 0\left|O_{\phi}\left(t, z, b_{\perp}, l\right)\right| \pi\left(P^{z}\right)\right\rangle e^{E_{\pi} t}
$$

with $E_{\pi}=\sqrt{m_{\pi}^{2}+P^{z 2}}$. The operator $O_{\phi}$ is defined as

$O_{\phi}\left(t, z, b_{\perp}, l\right) \equiv \bar{u}\left(t, z / 2, b_{\perp}\right) \Gamma_{\phi} W\left(z, b_{\perp}, l\right) d(t,-z / 2,0)$.

The quark fields $\bar{u}, d$ and Wilson link $W$ entering $O_{\phi}$ are all located at the same time slice $t$. $W$ has a staple shape and goes through spatial sites $(-z / 2,0) \rightarrow(-l, 0) \rightarrow$ $\left(-l, b_{\perp}\right) \rightarrow\left(z / 2, b_{\perp}\right)$. The Dirac matrix $\Gamma_{\phi}$ can be chosen as $\gamma_{5} \gamma_{0}$ or $\gamma_{5} \gamma_{3}$ so that $\Phi$ contains the LT contribution. Here, $\gamma_{i}(i=0,1,2,3)$ indicate the polarization direction $t, x, y, z$, respectively.

Up to $\mathcal{O}\left(\alpha_{s}\right)$ corrections, the hard kernel takes a simple form, denoted here as $H_{\Gamma}^{0}$. It can be obtained from a Fierz identity that $H_{\Gamma}^{0}=1 /\left(2 N_{c}\right)$ for $\Gamma=I, \gamma_{\perp}, \gamma_{5} \gamma_{\perp}$ and $-1 /\left(2 N_{c}\right)$ for $\Gamma=\gamma_{5}$ with $N_{c}=3$ the number of colors. Using $H_{\Gamma}^{0}$ as an input, one can further simplify the expression (2) as

$F_{\Gamma}\left(b_{\perp}, P^{z}\right) \underset{\text { LO kernel }}{\stackrel{P^{z} \rightarrow \infty}{=}} S\left(b_{\perp}, \mu\right) H_{\Gamma}^{0}\left|\phi\left(z=0, b_{\perp}, l=\infty, P^{z}\right)\right|^{2}$.

The soft function can be extracted by taking a ratio between $F_{\Gamma}$ and $H_{\Gamma}^{0}|\phi|^{2}$. When using Eq. (6), one always fixes $z=0$. Thus, in the following context, the variable $z$ is left out for simplicity.

Lattice setup.-We use the gauge ensemble of $N_{f}=$ $2+1+1$ clover-improved twisted mass fermions generated by the Extended Twisted Mass Collaboration [24]. In Eqs. (2) and (6), both $F_{\Gamma}$ and $\Phi$ contain the structure information of the pion, which is expected to be cancelled out at sufficiently large $P^{z}$, leaving the intrinsic soft function independent of either pion's structure or its mass. To check the mass dependence, we use four valence quark masses, corresponding to pion masses ranging 827 to $350 \mathrm{MeV}$. These valence quark masses together with other ensemble information are listed in Table I.

The three-point correlation function for the pion ME is

$$
\begin{aligned}
C_{\Gamma}^{3 p t}\left(b_{\perp}, P^{z}, t_{s}, t\right)= & \frac{1}{L^{3}} \sum_{\vec{x}} e^{-2 i P^{z} x_{z}} Z_{\Gamma}^{2} \\
& \times\left\langle O_{\pi}\left(t_{s},-P^{z}\right) \bar{u} \Gamma u\right. \\
& \left.\times\left(t, \vec{x}+b_{\perp}\right) \bar{d} \Gamma d(t, \vec{x}) O_{\pi}^{\dagger}\left(0, P^{z}\right)\right\rangle,
\end{aligned}
$$

with $t_{s}$ the source-sink separation. The operators $\bar{u} \Gamma u$ and $\bar{d} \Gamma d$ are inserted at time slice $t$ and constructed using the Coulomb-gauge-fixed-wall-source operators 
TABLE I. Ensemble parameters used in this work. We list the spatial and temporal extents, $L / a$ and $T / a$, the lattice spacing $a$, the sea quark mass $\mu_{\text {sea }}$, the pion mass $m_{\text {sea }}^{\pi}$, the number of measurements $N_{\text {meas }}=N_{\text {conf }} \times(T / 2)$, with $N_{\text {conf }}$ the number of configurations used, and four valence quark masses $\mu_{v i}$ for $i=0$, 1, 2, 3 together with the associated pion masses $m_{v i}^{\pi}$. All pion masses are given in units of $\mathrm{MeV}$.

\begin{tabular}{|c|c|c|c|c|c|c|c|}
\hline$L / a$ & $T / a$ & $a(\mathrm{fm})$ & & $a \mu_{\text {sea }}$ & $m_{\mathrm{sea}}^{\pi}$ & \multicolumn{2}{|c|}{$N_{\text {meas }}$} \\
\hline 24 & 48 & 0.093 & & 0.0053 & 350 & & $\times 24$ \\
\hline$a \mu_{v 0}$ & $m_{v 0}^{\pi}$ & $a \mu_{v 1}$ & $m_{v 1}^{\pi}$ & $a \mu_{v 2}$ & $m_{v 2}^{\pi}$ & $a \mu_{v 3}$ & $m_{v 3}^{\pi}$ \\
\hline 0.0053 & 350 & 0.013 & 545 & 0.018 & 640 & 0.03 & 827 \\
\hline
\end{tabular}

$$
O_{\pi}(t, \vec{P})=\sum_{\vec{x}, \vec{y}} \bar{u}(t, \vec{x}) \gamma_{5} d(t, \vec{y}) e^{-i \vec{P} \cdot \vec{y}}
$$

which are known to have a good overlap with the pion ground state. $Z_{\Gamma}$ is the renormalization factor to convert the bare lattice operator $\bar{q} \Gamma q$ to the renormalized one in the $\overline{\mathrm{MS}}$ scheme. The pion ME can be obtained from the connected part of three-point function at sufficiently large $t_{s}$ through

$$
C_{\Gamma}^{3 p t}\left(b_{\perp}, P^{z}, t_{s}, t\right)=\frac{\left|A_{w}\left(P^{z}\right)\right|^{2}}{(2 E)^{2}} e^{-E_{\pi} t_{s}} F_{\Gamma}\left(b_{\perp}, P^{z}\right),
$$

where $A_{w}\left(P^{z}\right)=L^{-(3 / 2)}\left\langle\pi\left(P^{z}\right)\left|O_{\pi}^{\dagger}\left(0, P^{z}\right)\right| 0\right\rangle$ is the overlap amplitude for the pion operator. According to parity, we have $A_{w}\left(P^{z}\right)=A_{w}\left(-P^{z}\right)$.

The correlation function for the quasi-TMDWF is constructed as

$C_{\Gamma_{\phi}}^{w f}\left(b_{\perp}, l, P^{z}, t\right)=\frac{Z_{\phi}}{L^{3}} \sum_{\vec{x}} e^{-i P^{z} x_{z}}\left\langle O_{\phi}\left(t, b_{\perp}, l\right) O_{\pi}^{\dagger}\left(0, P^{z}\right)\right\rangle$,

where $Z_{\phi}$ is the renormalization factor for the staple-shaped operator, which is found to be multiplicative $[25,26]$. We use $\Gamma_{\phi}=\gamma_{5} \gamma_{0}$ to avoid operator mixing for Wilson-type fermions in the renormalization procedure [26]. Stout smearing [27] has been widely used in the lattice calculations involving nonlocal operators to reduce ultraviolet fluctuations. Using up to 20 steps of smearing, studies [28-31] demonstrate that the physics is not altered. Here, we apply 5 steps of smearing to construct the operator $O_{\phi}\left(t, b_{\perp}, l\right)$. At large time separation $t$, one can extract the wave function $\phi$ via

$$
C_{\Gamma_{\phi}}^{w f}\left(b_{\perp}, l, P^{z}, t\right)=\frac{A_{w}\left(P^{z}\right)}{2 E_{\pi}} e^{-E_{\pi} t} \phi\left(b_{\perp}, l, P^{z}\right) .
$$

Combining Eqs. (9) and (11), the intrinsic soft function defined in Eq. (6) can be obtained through

$$
S\left(b_{\perp}\right)=\lim _{l \rightarrow \infty} \lim _{t_{s} \rightarrow \infty} \frac{C_{\Gamma}^{3 p t}\left(b_{\perp}, P^{z}, t_{s}, t\right)}{H_{\Gamma}^{0}\left|C_{\Gamma_{\phi}}^{w f}\left(b_{\perp}, l, P^{z}, \frac{t_{s}}{2}\right)\right|^{2}} .
$$

The lattice data show that $C_{\Gamma_{\phi}}^{w f}$ carries a small but nonvanishing imaginary part. In the determination of $S\left(b_{\perp}\right)$, we take into account the contributions from both the real and imaginary part of the wave function.

To examine the convergence of the lattice results at large momentum, we utilize 8 momenta with $P^{z}= \pm n(2 \pi / L)$ $(n=3,4,5,6)$, corresponding to a range from \pm 1.7 to $\pm 3.3 \mathrm{GeV}$. Given each $P^{z}$, we average the transition modes $\pi\left(P^{z}\right) \rightarrow \pi\left(-P^{z}\right)$ and $\pi\left(-P^{z}\right) \rightarrow \pi\left(P^{z}\right)$ and obtain a $15 \%-$ $20 \%$ reduction in the statistical error. For each momentum, we place the wall-source operator at every two time slices, which allows us to perform a time translation average for both $C_{\Gamma}^{3 p t}$ and $C_{\Gamma_{\phi}}^{w f}$. This helps to reduce the uncertainty of the soft function by nearly a factor of $\sqrt{T / 2}$.

Renormalization.-In our past calculation of the nucleon and Delta quasi-PDFs [31,32], we have utilized the regularization-independent momentum-subtraction (RI-MOM) scheme [33] developed for nonlocal operators [32,34]. The RI-MOM renormalized correlator is defined as $C_{\Gamma_{\phi}}^{w f, R I}=$ $C_{\Gamma_{\phi}}^{w f, b} Z_{\phi}^{R I}$, with the renormalization factor $Z_{\phi}^{R I}$ extracted by evaluating the amputated vertex functions with quark external states. This renormalization factor cancels the power and logarithmic divergences up to some systematic effects, such as discretization and HT effects. When using the stapleshaped operator, the systematic effects, which enter in the renormalization procedure, become more complicated. In previous LPC calculation [18] the renormalization is made through a ratio between the hadronic ME and the square root of the Wilson loop. As the latter is very different from the former, the systematic effects in the renormalization might be even larger than ones in the RI-MOM scheme. Later, Eq. (9) in Ref. [18] is used to define the renormalized soft function. This condition somehow limits the predictive power of the lattice calculation as the lattice results are forced to coincide with the perturbative ones at $b_{\perp, 0}=a$. In our calculation, we use the ratio scheme [19-21] instead, which has been proposed to replace the quark-state MEs in RI-MOM by the corresponding hadronic ones for a better control of systematics, such as discretization and HT effects. Here, we adopt the ratio scheme and construct the renormalized correlator as

$C_{\Gamma_{\phi}}^{w f, r}\left(b_{\perp}, l, P^{z}, t\right)=\frac{C_{\Gamma_{\phi}}^{w f, b}\left(b_{\perp}, l, P^{z}, t\right)}{C_{\Gamma_{\phi}}^{w f, b}\left(b_{\perp}, l, 0, t\right)} C_{\Gamma_{\phi} f, \overline{\mathrm{MS}}}(0,0,0, t)$,

where the bare correlators $C_{\Gamma_{\phi}}^{w f, b}\left(b_{\perp}, l, P^{z}, t\right)$ and $C_{\Gamma_{\phi}}^{w f, b}\left(b_{\perp}, l, 0, t\right)$ contain the same operator $O_{\phi}\left(t, b_{\perp}, l\right)$ and only differ by $P^{z}$. Thus, one can expect that a clean 
cancellation of UV divergences and other systematics can be achieved using the ratio scheme. Note that the renormalization has already been accomplished when taking the ratio $C_{\Gamma_{\phi}}^{w f, b}\left(b_{\perp}, l, P^{z}, t\right) / C_{\Gamma_{\phi}}^{w f, b}\left(b_{\perp}, l, 0, t\right)$. The coefficient $C_{\Gamma_{\phi}}^{w f, \overline{\mathrm{MS}}}(0,0,0, t)$ is introduced to restore the correct normalization at $b_{\perp} \rightarrow 0$. The conversion from the ratio scheme to the $\overline{\mathrm{MS}}$ scheme would require a perturbative calculation of the $\alpha_{s} \ln \left(b_{\perp} \mu\right)$ corrections. Up to these corrections one can treat $C_{\Gamma_{\phi}}^{w f, r}\left(b_{\perp}, l, P^{z}, t\right)$ and $C_{\Gamma_{\phi}}^{w f, \overline{\mathrm{MS}}}\left(b_{\perp}, l, P^{z}, t\right)$ as the same.

The renormalization for the local current operator $\bar{u} \Gamma u$ or $\bar{d} \Gamma d$ in $C_{\Gamma}^{3 p t}$ is straightforward. We find $Z_{S}^{\overline{\mathrm{MS}}}(2 \mathrm{GeV})=$ $0.641(3), Z_{P}^{\overline{\mathrm{MS}}}(2 \mathrm{GeV})=0.475(4)$ and $Z_{V}=0.712(2)$, $Z_{A}=0.753(3)$. Note that $Z_{V}$ and $Z_{A}$ are scheme and scale independent. These are calculated on dedicated $N_{f}=4$ ensembles with the same lattice action and spacing as the $N_{f}=2+1+1$ ensemble used for the MEs. The definitions of $Z_{V}, Z_{A}, Z_{S}$, and $Z_{P}$ follow the convention of Ref. [35]. Note that when the two operators $\bar{u} \Gamma u\left(b_{\perp}\right)$ and $\bar{d} \Gamma d(0)$ approach each other, a contact term appears and additional renormalization is required to match two bilinear quark operators to a local four-quark operator. Since the four-quark operators do not mix with any lower dimensional operators, we expect that additional renormalization effects are not large. It has been shown that renormalization factor for the four-quark operator only differs from that of the product of two local operators by $20 \%$ [36,37]. Such effects require further investigation but are not expected to alter the conclusions of this work.

Systematic effects.-In Eq. (3), the quasi-TMDWF is defined at an infinitely-large length of the Wilson line $l$. In a realistic lattice calculation, $l$ is truncated by a finite lattice size. At sufficiently large $l$, we find that the lattice results of $\left|C_{\Gamma_{\phi}}^{w f, r}\right|$ converge and yield a plateau for the region of $l \gtrsim 0.8 \mathrm{fm}$ (see the Supplemental Material [38]). Thus, fits to a constant lead to good $\chi^{2} /$ dof and provide the results of $\left|C_{\Gamma_{\phi}}^{w f, r}\right|$ at $l \rightarrow \infty$.

To extract reliably the pion ME from $\mid C_{\Gamma_{\phi} f, r}^{w f}$, the excitedstate contamination is another systematic effect to be controlled. We calculate the correlation functions at $t_{s} / a=6,8,10,12$ and use these data to perform a twostate fit. The lattice results are shown after removing the excited-state contamination.

After taking the extrapolation of $l \rightarrow \infty$ and examining the ground-state saturation at sufficiently large $t$, we use the simplified notation $C_{\Gamma_{\phi}}^{w f}\left(b_{\perp}, P^{z}\right)$ to replace $C_{\Gamma_{\phi}}^{w f}\left(b_{\perp}, l, P^{z}, t\right)$. To reveal the systematic effects more clearly, all figures presented in this work are compiled using the most precise lattice data at $m_{\pi}=827 \mathrm{MeV}$, unless specified otherwise.

Extraction of LT contribution-According to the proposal of Ref. [17], at $P^{z} \rightarrow \infty$, the same intrinsic soft function can be extracted from various pion MEs $F_{\Gamma}$ as far as $F_{\Gamma}$ contain the LT contribution. In this work, we make a complete investigation of the $\Gamma$ dependence of the soft function. Figure 1 (left) illustrates that the results of the soft function are significantly different when using various $F_{\Gamma}$ as inputs. Some results even carry the opposite sign.

To resolve this puzzle, we check the factorization in the LO perturbation theory and find at finite $P^{z}$

$$
\begin{aligned}
F_{\Gamma}\left(b_{\perp}, P^{z}\right)= & S\left(b_{\perp}\right) H_{\Gamma}^{0}\left|\phi\left(b_{\perp}, l, P^{z}\right)\right|^{2} \\
& +\sum_{\Gamma^{\prime} \neq \gamma_{5} \gamma_{0}, \gamma_{5} \gamma_{3}} S_{\Gamma^{\prime}}\left(b_{\perp}\right) H_{\Gamma \Gamma^{\prime}}^{0}\left|\phi_{\Gamma^{\prime}}\left(b_{\perp}, l, P^{z}\right)\right|^{2}+\cdots,
\end{aligned}
$$

where the factor $H_{\Gamma \Gamma^{\prime}}^{0}$ arises from Fierz rearrangement through

$\bar{u} \Gamma u\left(b_{\perp}\right) \bar{d} \Gamma d(0)=\sum_{\Gamma^{\prime}} H_{\Gamma \Gamma^{\prime}}^{0} \bar{u}\left(b_{\perp}\right) \Gamma^{\prime} d(0) \bar{d}(0) \Gamma^{\prime} u\left(b_{\perp}\right)$

with $H_{\Gamma \Gamma^{\prime}}^{0}=\left(1 / 16 N_{c}\right) \operatorname{Tr}\left(\Gamma \Gamma^{\prime} \Gamma \Gamma^{\prime}\right)$. The LT contribution carries a factor of $H_{\Gamma}^{0}$, which is the summation of $H_{\Gamma \Gamma^{\prime}}^{0}$ with $\Gamma^{\prime}=\gamma_{5} \gamma_{0}$ and $\gamma_{5} \gamma_{3}$. HT contributions enter in the second term of Eq. (14) with the wave function $\phi_{\Gamma^{\prime}}\left(b_{\perp}, l, P^{z}\right)=$ $\left\langle 0\left|\bar{u}\left(b_{\perp}\right) \Gamma^{\prime} W\left(b_{\perp}, l\right) d(0)\right| \pi\left(P^{z}\right)\right\rangle$. Although HT contributions are expected to be much smaller than the LT one at sufficiently large momentum, in a realistic lattice calculation, where the typical size of $P^{z}$ is a few $\mathrm{GeV}$, the contamination from HT may be significant. We find that the lattice result of $\phi_{\Gamma^{\prime}}$ for $\Gamma^{\prime}=\gamma_{5}$ is even larger than the LT $\phi$. Such large HT contamination explains why some $F_{\Gamma}\left(b_{\perp}, P^{z}\right)$ carry the opposite sign, as observed in Fig. 1. Here we focus on the largest power corrections associated with $\phi_{\Gamma^{\prime}}$. Any residual corrections are represented by the ellipsis in Eq. (14).

Note that in Fig. 1, results at the largest momentum $P^{z}=6(2 \pi / L) \approx 3.3 \mathrm{GeV}$ are presented. When $P^{z}$ decreases, the situation becomes even worse. This is not surprising, as LT contributions are enhanced at large $P^{z}$. Considering the fact that the $P^{z}$ values accessible on the lattice are quite limited, we draw the conclusion that it is essential to remove the HT effects in the calculation of the soft function. Here we take two steps. (i) First, we calculate $\phi_{\Gamma^{\prime}}$ with various $\Gamma^{\prime}$ and then pick up all $\phi_{\Gamma^{\prime}}$ with relatively large size. It leads to four $\phi_{\Gamma^{\prime}}$ with $\Gamma^{\prime}=\gamma_{5}, \sigma_{02}, \sigma_{12}, \sigma_{23}$. (ii) Second, we define improved pion MEs as $\sum_{\Gamma} c_{\Gamma} F_{\Gamma}\left(b_{\perp}, P^{z}\right)$, where the coefficients $c_{\Gamma}\left(\Gamma=I, \gamma_{5}, \gamma_{\perp}, \gamma_{5} \gamma_{\perp}\right)$ are chosen appropriately to cancel contributions from $\phi_{\Gamma^{\prime}}\left(\Gamma^{\prime}=\gamma_{5}, \sigma_{02}, \sigma_{12}, \sigma_{23}\right)$.

Following the above steps, we finally obtain five improved pion MEs as a simple combination of two $F_{\Gamma}\left(b_{\perp}\right)$, namely,

$$
\begin{array}{lll}
\frac{1}{2}\left(F_{\gamma_{5} \gamma_{1}}+F_{\gamma_{1}}\right), & \frac{1}{2}\left(F_{\gamma_{5} \gamma_{2}}+F_{\gamma_{2}}\right), & \frac{1}{2}\left(-F_{\gamma_{5}}+F_{\gamma_{5} \gamma_{2}}\right), \\
\frac{1}{2}\left(-F_{\gamma_{5}}+F_{I}\right), & \frac{1}{2}\left(F_{I}+F_{\gamma_{2}}\right) . &
\end{array}
$$




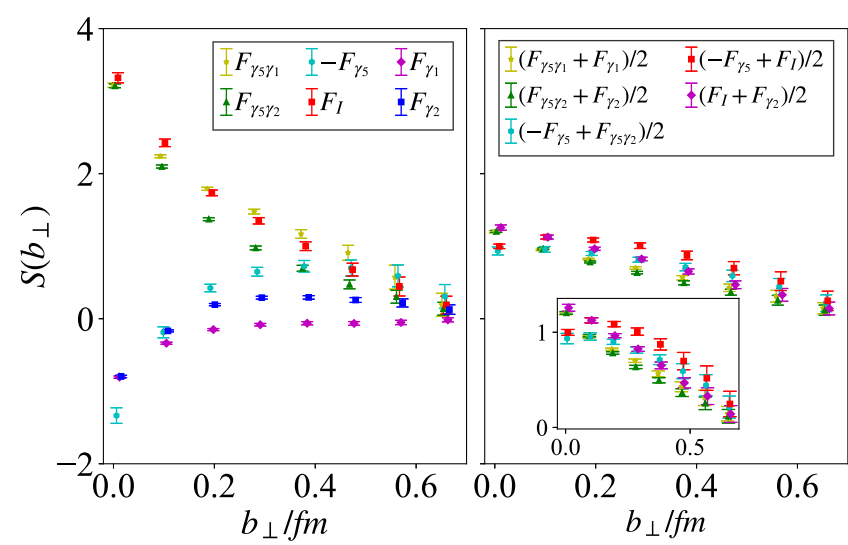

FIG. 1. The intrinsic soft function $S\left(b_{\perp}\right)$ as a function of transverse separation $b_{\perp}$ at $P^{z}=6(2 \pi / L) \approx 3.3 \mathrm{GeV}$ and $m_{\pi}=827 \mathrm{MeV}$. On the left panel, $S\left(b_{\perp}\right)$ are compiled using the pion MEs $F_{\Gamma}$ as inputs, with $\Gamma=I, \gamma_{5}, \gamma_{\perp}, \gamma_{5} \gamma_{\perp}$. For $\gamma_{\perp}$, there are two choices: $\gamma_{1}$ parallel to $b_{\perp}$ and $\gamma_{2}$ perpendicular to $b_{\perp}$. On the right panel, $S\left(b_{\perp}\right)$ are compiled using the improved pion MEs, where the large HT contamination has been canceled significantly and the results show much better consistency.

Figure 1 (right) shows the soft function compiled using the five improved pion MEs. By canceling the dominant HT effects, the results become much more consistent. Residual deviations serve as measure of important systematic effects to be controlled in future studies.

Results of the soft function.-After checking the consistency among the various improved pion MEs, we use the choice of $\frac{1}{2}\left(F_{\gamma_{5} \gamma_{1}}+F_{\gamma_{1}}\right)$ as an example to present the results of $S\left(b_{\perp}\right)$ for various momenta $P^{z}$ and pion masses $m_{v i}^{\pi}$.

In Fig. 2, $S\left(b_{\perp}, P^{z}\right)$ is shown together with the one-loop perturbative curve [39],

$$
S_{\overline{\mathrm{MS}}}\left(b_{\perp}, \mu\right)=1-\frac{\alpha_{s} C_{F}}{\pi} \ln \frac{\mu^{2} b_{\perp}^{2}}{4 e^{-2 \gamma_{E}}}+\mathcal{O}\left(\alpha_{s}^{2}\right),
$$

where one-loop and four-loop values of $\alpha_{s}$ are used at the physically most relevant scale of $S\left(b_{\perp}\right)$, i.e., $1 / b_{\perp}$. The

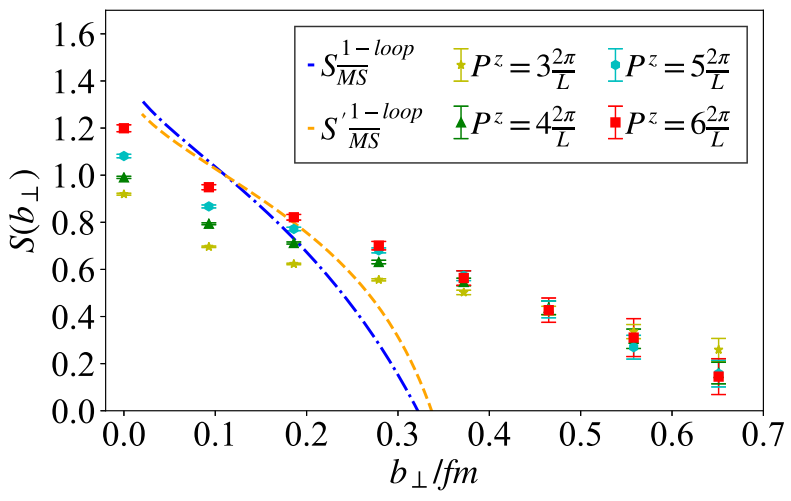

FIG. 2. The lattice results of $S\left(b_{\perp}\right)$ for various momenta at $m_{\pi}=827 \mathrm{MeV}$, together with the one-loop perturbative result $S_{\overline{\mathrm{MS}}}^{1-\text { loop }}$ and its variant $S_{\overline{\mathrm{MS}}}^{1-\text { loop }}$ with $\alpha_{s}$ including up to 4 loops. The scale $\mu$ in Eq. (17) is set as $\mu=2 \mathrm{GeV}$. scale $\mu$ is set as $\mu=2 \mathrm{GeV}$. We note that the lattice results agree qualitatively with the perturbative function at around $b_{\perp} \sim 0.2 \mathrm{fm}$, particularly at the largest boost and when higher-order effects are partially included via $\alpha_{s}$. At larger $b_{\perp}$, nonperturbative features start to set in and the decay of $S\left(b_{\perp}\right)$ is slower than the perturbative prediction. It is also noteworthy that the convergence of the lattice results in $P^{z}$ clearly increases with $b_{\perp}$-the results from the two largest $P^{z}$ are compatible for $b_{\perp} \gtrsim 0.2 \mathrm{fm}$, while smaller transverse separations will need yet larger boosts to establish convergence.

In Fig. 3, we examine the pion mass dependence of the soft function. Although $S\left(b_{\perp}\right)$ is extracted from pion MEs which depend on the detailed process of $\pi\left(P^{z}\right) \rightarrow \pi\left(-P^{z}\right)$, the factorization allows us to cancel this process dependence. Performing the calculation at four pion masses, we find that the lattice results are generally consistent within statistical errors, although a small systematic increase is found when decreasing $m_{\pi}$. Within current errors, this observation is consistent with the expectation from factorization theory [17] that the soft function should not depend on the detailed hadronic information from the initial or final state.

Results for the Collins-Soper kernel.-The Collins-Soper kernel $K\left(b_{\perp}, \mu\right)$ governs the rapidity evolution of the TMDPFs. In LaMET, the quasi-TMDPDF is factorized into the light-cone TMDPDF and a $K\left(b_{\perp}, \mu\right) \ln \left(\zeta^{z} / \zeta\right)$ factor, where $\zeta^{z}=2\left(x P^{z}\right)^{2}$, with $P^{z}$ playing the role of the rapidity, while $\zeta$ is the light-cone counterpart of $\zeta^{z}[40]$. Thus, by taking the ratio of quasi-TMDPDFs at different values of $P^{z}$, one can extract $K\left(b_{\perp}, \mu\right)$. This ratio can also be expressed in terms of the quasi-TMDWFs [18] as

$$
\begin{aligned}
K\left(b_{\perp}, \mu\right) & =\lim _{l \rightarrow \infty} \frac{1}{\ln \left(P_{1}^{z} / P_{2}^{z}\right)} \ln \left|\frac{\phi\left(b_{\perp}, l, P_{1}^{z}\right) / E_{1}}{\phi\left(b_{\perp}, l, P_{2}^{z}\right) / E_{2}}\right| \\
& =\frac{1}{\ln \left(P_{1}^{z} / P_{2}^{z}\right)} \ln \left|\frac{C_{\Gamma_{\phi}}^{w f}\left(b_{\perp}, P_{1}^{z}\right)}{C_{\Gamma_{\phi}}^{w f}\left(b_{\perp}, P_{2}^{z}\right)} \frac{C_{\Gamma_{\phi}}^{w f}\left(0, P_{2}^{z}\right)}{C_{\Gamma_{\phi}}^{w f}\left(0, P_{1}^{z}\right)}\right| .
\end{aligned}
$$

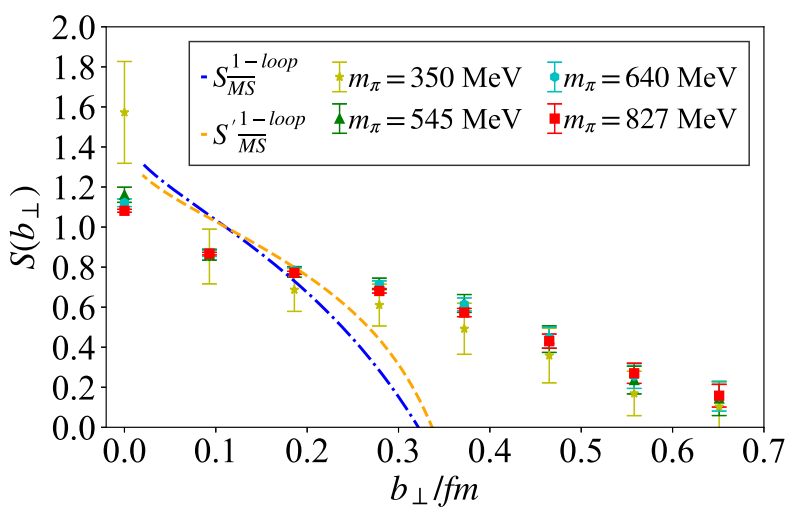

FIG. 3. The intrinsic soft function $S\left(b_{\perp}\right)$ for the pion masses ranging from 827 to $350 \mathrm{MeV}$. Here, we show results calculated at $P^{z}=5(2 \pi / L)$ as an example. 


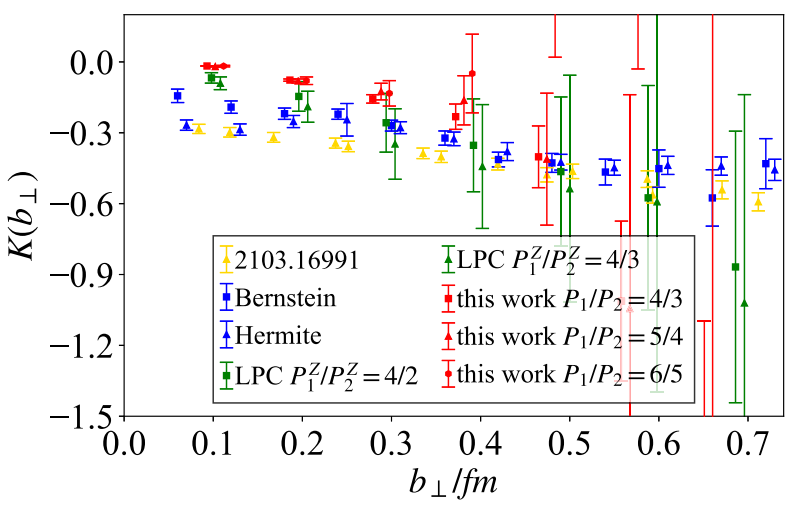

FIG. 4. The lattice results for the Collins-Soper kernel $K\left(b_{\perp}, \mu\right)$ from various calculations, described by yellow [23], blue [22], green [18], and red. Results from the same calculation are shifted horizontally to make an easier comparison. For this work, the setup at $m_{\pi}=827 \mathrm{MeV}$ is used.

In Fig. 4, the lattice results of $K\left(b_{\perp}, \mu\right)$ from this work are shown together with data from other calculations. The results exhibit similar dependence on $b_{\perp}$ with some discrepancies, which indicate unquantified systematics. Both the LPC results and ours are calculated using the quasi-TMDWFs as inputs. Thus, it is not surprising that these results are in better agreement.

Conclusion.-Within the framework of lattice QCD we calculate the intrinsic soft function introducing a number of crucial steps that enable its reliable extraction. Our work adds evidence that the methodology proposed in Ref. [17] is indeed suitable for the determination of these quantities. There is room for further improvements. For example, only the LO perturbative hard kernel is used in this calculation and future work needs to examine higher-order corrections. On the lattice side, several sources of systematics need to be addressed, including e.g., cutoff effects and further investigation of quark mass dependence towards the physical one. Nevertheless, this methodology coupled with the improvements introduced in this work, requiring synergy of perturbative and lattice QCD, is shown to be very promising and can provide important first-principle insights into TMD hadron structure.

We thank Lu-Chang Jin, Yi-Zhuang Liu, Yu-Sheng Liu, Yan-Qing Ma, Wei Wang, Yi-Bo Yang, Qi-An Zhang, and Yong Zhao for valuable discussions. We thank Maximilian Schlemmer, Qi-An Zhang, and Yong Zhao for providing their data of the Collins-Soper kernel. X. F. and S. C. X. are supported in part by NSFC of China under Grants No. 12125501, No. 12141501, and No. 11775002 and National Key Research and Development Program of China under Contracts No. 2020YFA0406400. X. F. and C. L. are supported in part by NSFC of China under Grant No. 12070131001. Y. L. and C. L. are supported in part by CAS Interdisciplinary Innovation Team and NSFC of China under Grant No. 11935017. F. S. was funded by the NSFC and the Deutsche Forschungsgemeinschaft
(DFG, German Research Foundation) through the funds provided to the Sino-German Collaborative Research Center TRR110 "Symmetries and the Emergence of Structure in QCD" (NSFC Grant No. 12070131001, DFG Project-ID 196253076-TRR 110). K. C. is supported by the National Science Centre (Poland) grant SONATA BIS No. 2016/22/E/ST2/00013. K. H. is financially supported by the Cyprus Research and Innovation Foundation under Contract NO. POST-DOC/0718/0100. M. C. and A. S. acknowledge financial support by the U.S. Department of Energy, Office of Nuclear Physics, Early Career Award under Grant No. DE-SC0020405. J. T. acknowledges support from project NextQCD, co-funded by the European Regional Development Fund and the Republic of Cyprus through the Research and Innovation Foundation (EXCELLENCE/0918/0129). The calculation was carried out on TianHe-3 (prototype) at Chinese National Supercomputer Center in Tianjin. This work also used computational resources from the John von NeumannInstitute for Computing on the Juwels booster system at the research center in Juelich, under the project with id ECY00 and on the Cyclone machine of the Cyprus Institute under project ID pro21a106.

* Corresponding author.

xu.feng@pku.edu.cn

[1] G. Parisi and R. Petronzio, Nucl. Phys. B154, 427 (1979).

[2] I. Scimemi and A. Vladimirov, J. High Energy Phys. 06 (2020) 137.

[3] J. Collins, L. Gamberg, A. Prokudin, T. C. Rogers, N. Sato, and B. Wang, Phys. Rev. D 94, 034014 (2016).

[4] P. J. Mulders and R. D. Tangerman, Nucl. Phys. B461, 197 (1996); B484, 538(E) (1997).

[5] R. Abdul Khalek et al., arXiv:2103.05419.

[6] D. P. Anderle et al., Front. Phys. 16, 64701 (2021).

[7] J. C. Collins, D. E. Soper, and G. F. Sterman, Nucl. Phys. B250, 199 (1985).

[8] J. C. Collins, D. E. Soper, and G. F. Sterman, Nucl. Phys. B308, 833 (1988).

[9] X.-d. Ji, J.-p. Ma, and F. Yuan, Phys. Rev. D 71, 034005 (2005).

[10] X.-d. Ji, J.-P. Ma, and F. Yuan, Phys. Lett. B 597, 299 (2004).

[11] J. Collins and T. C. Rogers, Phys. Rev. D 96, 054011 (2017).

[12] M. G. Echevarria, I. Scimemi, and A. Vladimirov, Phys. Rev. D 93, 054004 (2016).

[13] Y. Li and H. X. Zhu, Phys. Rev. Lett. 118, 022004 (2017).

[14] X. Ji, Y.-S. Liu, Y. Liu, J.-H. Zhang, and Y. Zhao, Rev. Mod. Phys. 93, 035005 (2021).

[15] X. Ji, Phys. Rev. Lett. 110, 262002 (2013).

[16] X. Ji, Sci. China Phys. Mech. Astron. 57, 1407 (2014).

[17] X. Ji, Y. Liu, and Y.-S. Liu, Nucl. Phys. B955, 115054 (2020).

[18] Q.-A. Zhang, J. Hua, Y. Huo, X. Ji, Y. Liu, Y. S. Liu, M. Schlemmer, A. Schafer, P. Sun, W. Wang, and Y. B. Yang (Lattice Parton Collaboration), Phys. Rev. Lett. 125, 192001 (2020). 
[19] K. Orginos, A. Radyushkin, J. Karpie, and S. Zafeiropoulos, Phys. Rev. D 96, 094503 (2017).

[20] T. Izubuchi, X. Ji, L. Jin, I. W. Stewart, and Y. Zhao, Phys. Rev. D 98, 056004 (2018).

[21] X. Gao, L. Jin, C. Kallidonis, N. Karthik, S. Mukherjee, P. Petreczky, C. Shugert, S. Syritsyn, and Y. Zhao, Phys. Rev. D 102, 094513 (2020).

[22] P. Shanahan, M. Wagman, and Y. Zhao, Phys. Rev. D 102, 014511 (2020).

[23] M. Schlemmer, A. Vladimirov, C. Zimmermann, M. Engelhardt, and A. Schäfer, J. High Energy Phys. 08 (2021) 004.

[24] C. Alexandrou, S. Bacchio, P. Charalambous, P. Dimopoulos, J. Finkenrath, R. Frezzotti, K. Hadjiyiannakou, K. Jansen, G. Koutsou, B. Kostrzewa, M. Mangin-Brinet, G. Rossi, S. Simula, and C. Urbach, Phys. Rev. D 98, 054518 (2018).

[25] M. A. Ebert, I. W. Stewart, and Y. Zhao, J. High Energy Phys. 03 (2020) 099.

[26] M. Constantinou, H. Panagopoulos, and G. Spanoudes, Phys. Rev. D 99, 074508 (2019).

[27] C. Morningstar and M. J. Peardon, Phys. Rev. D 69, 054501 (2004).

[28] C. Alexandrou, K. Cichy, M. Constantinou, K. Jansen, A. Scapellato, and F. Steffens, Phys. Rev. Lett. 121, 112001 (2018).

[29] C. Alexandrou, K. Cichy, M. Constantinou, K. Jansen, A. Scapellato, and F. Steffens, Phys. Rev. D 98, 091503 (2018).
[30] C. Alexandrou, K. Cichy, M. Constantinou, K. Hadjiyiannakou, K. Jansen, A. Scapellato, and F. Steffens, Phys. Rev. D 99, 114504 (2019).

[31] Y. Chai, Y. Li, S. Xia, C. Alexandrou, K. Cichy, M. Constantinou, X. Feng, K. Hadjiyiannakou, K. Jansen, G. Koutsou, C. Liu, A. Scapellato, and F. Steffens, Phys. Rev. D 102, 014508 (2020).

[32] C. Alexandrou, K. Cichy, M. Constantinou, K. Hadjiyiannakou, K. Jansen, H. Panagopoulos, and F. Steffens, Nucl. Phys. B923, 394 (2017).

[33] G. Martinelli, C. Pittori, C. T. Sachrajda, M. Testa, and A. Vladikas, Nucl. Phys. B445, 81 (1995).

[34] M. Constantinou and H. Panagopoulos, Phys. Rev. D 96, 054506 (2017).

[35] C. Alexandrou, M. Constantinou, and H. Panagopoulos (ETM Collaboration) Phys. Rev. D 95, 034505 (2017).

[36] N. H. Christ, T. Izubuchi, C. T. Sachrajda, A. Soni, and J. Yu (RBC, UKQCD Collaborations), Phys. Rev. D 88, 014508 (2013).

[37] Y. Aoki, R. Arthur, T. Blum, P. A. Boyle, D. Brommel et al. (RBC, UKQCD Collaborations), Phys. Rev. D 83, 074508 (2011).

[38] See Supplemental Material at http://link.aps.org/supplemental/ 10.1103/PhysRevLett.128.062002 for the convergence of the quasi-TMDWF at a sufficiently large length of the Wilson line.

[39] X. Ji, J.-P. Ma, and F. Yuan, Phys. Rev. D 71, 034005 (2005).

[40] M. A. Ebert, I. W. Stewart, and Y. Zhao, Phys. Rev. D 99, 034505 (2019). 